\title{
Self-Assembly of Patchy Colloidal Rods into Photonic Crystals Robust to Stacking Faults
}

Andreas Neophytou, ${ }^{\dagger}$ Vinothan N. Manoharan, ${ }^{\dagger, \uparrow}$ and Dwaipayan Chakrabarti*,†

†School of Chemistry, University of Birmingham, Edgbaston, Birmingham B15 2TT, UK

$\ddagger$ Harvard John A. Paulson School of Engineering and Applied Sciences, Harvard

University, Cambridge, Massachusetts 02138, USA

\Department of Physics, Harvard University, Cambridge, Massachusetts 02138, USA

E-mail: d.chakrabarti@bham.ac.uk

Supporting Information 


\section{Monte Carlo annealing protocols}

We performed a series of five independent Monte Carlo simulations with three systems of $N=$ 2000 AB-triblock patchy rods (TPRs) corresponding to different values of the aspect ratio $L^{*}=\{0,0.5,1\}$. For each independent run, we followed the following annealing protocols: $T^{*}=1.0 \rightarrow 0.6$ with $\Delta T^{*}=-0.2 ; T^{*}=0.5 \rightarrow 0.4$ with $\Delta T^{*}=-0.1 ; T^{*}=0.38 \rightarrow 0.30$ with $\Delta T^{*}=-0.02 ; T^{*}=0.25 \rightarrow 0.20$ with $\Delta T^{*}=-0.05 ; T^{*}=0.18 \rightarrow 0.16$ with $\Delta T^{*}=-0.02$, with 1.5 million Monte Carlo cycles run for temperatures between [0.5-1.0] and 3 million Monte Carlo cycles run for temperatures between $[0.18-0.4]$. For the $L^{*}=0$ system, 10 million Monte Carlo cycles were run at $T^{*}=0.16$, followed by 3 million Monte Carlo cycles for $T^{*}=0.14 \rightarrow 0.1$ with $\Delta T^{*}=-0.02$. For the $L^{*}=0.5$ system, 3 million Monte Carlo cycles were run at $T^{*}=0.16$, followed by 10 million Monte Carlo cycles at $T^{*}=0.14$, and then 3 million Monte Carlo Cycles were run at $T^{*}=0.12$ and 0.10 . For the $L^{*}=1$ system, 3 million Monte Carlo cycles were run for each temperature in the range $T^{*}=0.16 \rightarrow 0.14$ with $\Delta T^{*}=-0.02 ; 10$ million Monte Carlo cycles were performed at $T^{*}=0.12$, followed by 3 million Monte Carlo cycles at $T^{*}=0.11$ and 0.10 .

We also carried out a series of five independent Monte Carlo simulations with a system of $N=2000$ AA-TPRs, for $L^{*}=0.5$. The following annealing protocol was followed: $T^{*}=1.0 \rightarrow 0.6$ with $\Delta T^{*}=-0.2 ; T^{*}=0.5 \rightarrow 0.4$ with $\Delta T^{*}=-0.1 ; T^{*}=0.35 \rightarrow 0.2$ with $\Delta T^{*}=-0.05 ; T^{*}=0.18 \rightarrow 0.14$ with $\Delta T^{*}=-0.02 ; T^{*}=0.13 \rightarrow 0.10$ with $\Delta T^{*}=-0.01$. 1, 3 and 5 million Monte Carlo cycles were run at each temperature between [0.2-1.0], [0.13$0.18]$ and [0.10-0.12], respectively.

In the case of the $\mathrm{AA}^{\prime}-\mathrm{TPR}$, a system of $N=2000$ particles with $L^{*}=0.5$ was equilibrated at $T^{\star}=1$ by performing 1 million Monte Carlo cycles. The system was then subject to one-step cooling from $T^{*}=1$ to a temperature that was guided by the temperature at which the crystallization was observed in our simulations of the AB system and several short simulations with the $\mathrm{AA}^{\prime}$ system. This led to one-step cooling from $T^{*}=1 \rightarrow 0.118$ where 25 million Monte Carlo cycles were performed during which crystallization was observed. 


\section{Programming self-limiting tetrahedral clusters}

The hierarchical self-assembly scheme for AB-triblock patchy rods (TPRs), laid out in Figure 1a, is critically reliant upon the exclusive formation of tetrahedral clusters in the first stage of assembly before the second stage of assembly takes place. ${ }^{113}$ Here, we have exploited the width of the patches to satisfy a geometric criterion so that the formation of tetrahedra is favored over higher coordination clusters, such as octahedra. In the model used to describe the patch-patch interactions between TPRs, these higher coordination clusters are not prohibited; however, they can be made energetically disfavored by tuning the geometry of the patches.

Supporting Figure 1 shows the distance and angular dependence of the TPR pair potential for the parameter $s$ set to $s=5$, and the patch half-angles set to $\delta=80^{\circ}$. For this set of parameters, the particles cannot interact across the basal plane of octahedral clusters. This ensures that the particles form only one additional bond in octahedra as compared to tetrahedra. Additionally, it is apparent that the pair potential is minimized when the angle $\theta$, as shown in Supporting Figure 1, is less than a "critical" angle $\left(\theta_{\mathrm{c}} \sim 20^{\circ}\right)$. Above this critical angle, bonds can still form between the particles as long as $\theta \leq \delta$. However, the bonds become energetically less favorable as this angle increases. The value of this critical angle depends on the patch width $\delta$ and can thus be tuned by altering the geometry of the patches. Due to the difference in the relative orientation of the particles in tetrahedra and octahedra, the patch-patch bonds between TPRs in a tetrahedral cluster are energetically more favorable than those in an octahedral cluster for $s=5$ and $\delta=80^{\circ}$.

Supporting Figure 2 shows the potential energy per particle for various clusters as a function of $\delta$. It is evident that these higher coordination clusters are indeed energetically disfavored when $\delta=80^{\circ}$. Additionally, we expect that particles in a tetrahedral cluster will have greater rotational entropy than particles in an octahedral cluster, further favoring the formation of tetrahedra over octahedra. ${ }^{415}$ Therefore, we are able to design a system of TPRs to reliably self-assemble into structures with tetrahedral coordination by correctly 
choosing the geometry of the patches and the range of patch-patch interactions. By setting $\delta=80^{\circ}$ a sizeable stabilization of tetrahedra over octahedra is provided, while also ensuring a large energetic driving force for the formation of tetrahedra over trigonal planar clusters, which can be expected to form in significant numbers for narrow patch half-angles. ${ }^{6}$ It is of interest to note that in the Kern-Frenkel potential, widely used to model the interactions between spherical triblock patchy particles, the patch-patch interactions are either "on" or "off" depending on the distance between two particles and their relative orientation.7 Therefore, in the Kern-Frenkel model, the competition from higher coordination clusters, such as octahedra, can be ruled out altogether by limiting the width of the patches so that only tetrahedral cluster are formed at an optimal condition. $\frac{4|6| 8}{46}$

In Supporting Figure 5, we show the evolution of cluster distributions with temperature for systems of AB-TPRs with aspect ratios of $L^{*}=0,0.5,1$ and $\delta=80^{\circ}$. We find that at high temperatures there are predominantly dimers and trimers; the number of tetramers gradually increases as the temperature decreases, until practically only tetramers are present. We have also established in Figure $2 \mathrm{~b}$ that these tetramers are tetrahedral clusters. This same trend was recently observed for a model system of one-patch Janus colloids, which was also found to be in good agreement with experiments. ${ }^{6}$ Additionally, this general trend in the relative populations of different clusters with temperature is common to all three systems considered. We therefore infer that the geometry of the patches sitting on the spherical caps is the principal factor, along with the range of patch-patch interactions, driving the selfassembly of the particles into self-limiting tetrahedral clusters, which occurs at an optimal temperature window governed by the energetics of patch-patch interactions. This inference is further reinforced by Supporting Figure 4, where we find that the lattice energy of the segmented rod-connected diamond (sRCD) structures is invariant to the aspect ratio of the particles, but does depend on the patch half-angle. 


\section{Self-assembly of triblock patchy rods with polydisperse patch half- angles}

In order to assess the robustness of our design principles to imperfection to the fabrication of triblock patchy rods (TPRs), we investigated the self-assembly of TPRs with polydisperse patch half-opening angles. In Ref. [8], Chaudhary et al. were able to synthesize a monodisperse system of colloidal Janus matchsticks with a patch angle distribution of $2 \delta=116^{\circ} \pm 12^{\circ}$. We therefore studied three systems of AB-TPRs, each having a Gaussian distribution for patch half-angles with the standard deviation $\sigma_{\delta}=5^{\circ}$, around the mean values of $\bar{\delta}=70^{\circ}, 80^{\circ}, 90^{\circ}$. Hereafter, we will refer to these systems as $\mathrm{AB}_{1}, \mathrm{AB}_{2}$ and $\mathrm{AB}_{3}$, respectively. All three systems contained $N=1000$ particles having an aspect ratio of $L^{*}=0.5, \rho^{*}=0.241, \epsilon_{\mathrm{AA}}=5 \epsilon_{\mathrm{BB}}$ and $\epsilon_{\mathrm{AB}}=\sqrt{\epsilon_{\mathrm{AA}} \epsilon_{\mathrm{BB}}}$.

Supporting Figure 8 shows the self-assembly of AB-TPRs with polydisperse patch angles when subjected to gradual cooling in Monte Carlo simulations and the corresponding distributions of patch half-angles. We find that, as observed in the case for monodisperse patches with the half-angle of $\delta=80^{\circ}$, these systems gradually transition from a fluid phase composed primarily of dimers and trimers at high temperatures, to a tetrahedral cluster phase at lower temperatures. However, we also find that for the $\mathrm{AB}_{1}$ system there are trimers in a sizeable number at the end of the first stage of assembly, which is to be expected for a system of patchy particles with narrow patches that still allow for three contacts to form. ${ }^{6}$ In contrast, there is a small population of pentamers in the $\mathrm{AB}_{3}$ system, showing that higher coordination clusters begin to become more prevalent as the size of the patches increases. These observations reinforce the importance of optimally designing the geometry of the patches in order to program the self-assembly of the particles into a phase of self-limiting tetrahedral clusters, and that there exists a "Goldilocks" window of patch half-angles that sufficiently stabilizes tetrahedral clusters over trigonal planar clusters and higher coordination clusters.

Following the first stage of assembly, only the $\mathrm{AB}_{2}$ system crystallizes into a segmented rod-connected diamond (s-RCD) structure, as shown in Supporting Figures 8 and 9. We 
find that the $A_{1}$ system struggles to form the emergent vertex-sharing tetrahedra, which drive crystallization in the second stage of assembly. Instead, the system becomes arrested in an amorphous structure, as shown in Supporting Figure 9a. This inability of the $\mathrm{AB}_{1}$ system to crystallize is further exacerbated by the presence of persistent trimers following the first stage assembly. Unlike the $\mathrm{AB}_{2}$ system (see Supporting Figure $9 \mathrm{~b}$ ), the $\mathrm{AB}_{3}$ system also fails to crystallize; however, the reason for this failure is different from that for the $\mathrm{AB}_{1}$ system. Supporting Figure $9 \mathrm{c}$ shows that the $\mathrm{AB}_{3}$ system fails to crystallize due to the preferential formation of distorted trigonal bipyramidal motifs over the vertex-sharing tetrahedra required for the s-RCD crystals. These motifs are formed via vertex-face bonds between the tetrahedral clusters that are facilitated by patch A-patch B interactions. Due to the wider patches in the $\mathrm{AB}_{3}$ system, and the fact that we set the strength of the interactions such that $\epsilon_{\mathrm{BB}}<\epsilon_{\mathrm{AB}}<\epsilon_{\mathrm{AA}}$, it is more favorable for the system to form the three distorted AB-bonds of the trigonal bipyramidal motifs than the three BB-bonds of the desired vertexsharing tetrahedra.

We performed a corresponding set of simulations for the $\mathrm{AA}^{\prime}$ system, with one-step cooling from $T^{*}=1$. Again, we considered three systems, each having a Gaussian distribution for patch half-angles with the standard deviation $\sigma_{\delta}=5^{\circ}$, around the mean values of $\bar{\delta}=70^{\circ}, 80^{\circ}, 90^{\circ}$. Hereafter, these systems are referred to as $\mathrm{AA}_{1}^{\prime}, \mathrm{AA}_{2}^{\prime}$ and $\mathrm{AA}_{3}^{\prime}$, respectively. All three systems contained $N=1000$ particles having an aspect ratio of $L^{*}=0.5$, $\rho^{*}=0.241, \epsilon_{\mathrm{AA}}=\epsilon_{\mathrm{BB}}=1$ and $\epsilon_{\mathrm{AB}}=0$. We find that, like the $\mathrm{AB}_{1}$ system, the $\mathrm{AA}_{1}^{\prime}$ system is unable to crystallize at any of the temperatures considered $\left(T^{*}=0.08,0.09,0.10,0.11\right)$ as it struggles to form the emergent vertex-sharing tetrahedra due to the narrower patch angles. However, we observe that both the $\mathrm{AA}_{2}^{\prime}$ and $\mathrm{AA}_{3}^{\prime}$ systems crystallize at $T^{*}=0.118$ and $T^{*}=0.13$, respectively. Supporting Figure 12 shows the nucleation of the crystal in the $\mathrm{AB}_{3}$ system from the fluid. As observed in the case for monodisperse patches with half-angles of $\delta=80^{\circ}$, we find that both the $\mathrm{AA}_{2}^{\prime}$ and $\mathrm{AA}_{3}^{\prime}$ systems crystallize via two-step nucleation pathways, with the first step involving the rapid formation of a fluid of tetrahedral clusters. 


\section{Supporting Figures}


Supporting Figure 1: Triblock patchy rod model.

(a) Two-dimensional representation of the triblock patchy rod (TPR) model, where $L$ is the length of the line segment (thick black line) that connects the centers of the spherocylinder caps, and $\sigma$ is the diameter of the spherocylinder. Attractive circular patches, distinguished by color-coding, are placed on opposite poles. The sizes of the patches, characterized by the half-opening angles $\alpha$ and $\beta$, and strengths of the interactions between the patches can vary. The effective pair potential as a function of $(\mathbf{b})$ the center-to-center distance $(r)$ and $(\mathbf{c})$ the angle $\theta$ for one TPR shown in the inset, while the corresponding angle for the other TPR is set to $\theta_{\min }$, where $r_{\min }=\sqrt[50]{2} \sigma$ and $\theta_{\min }=0^{\circ} \cdot 7$ 

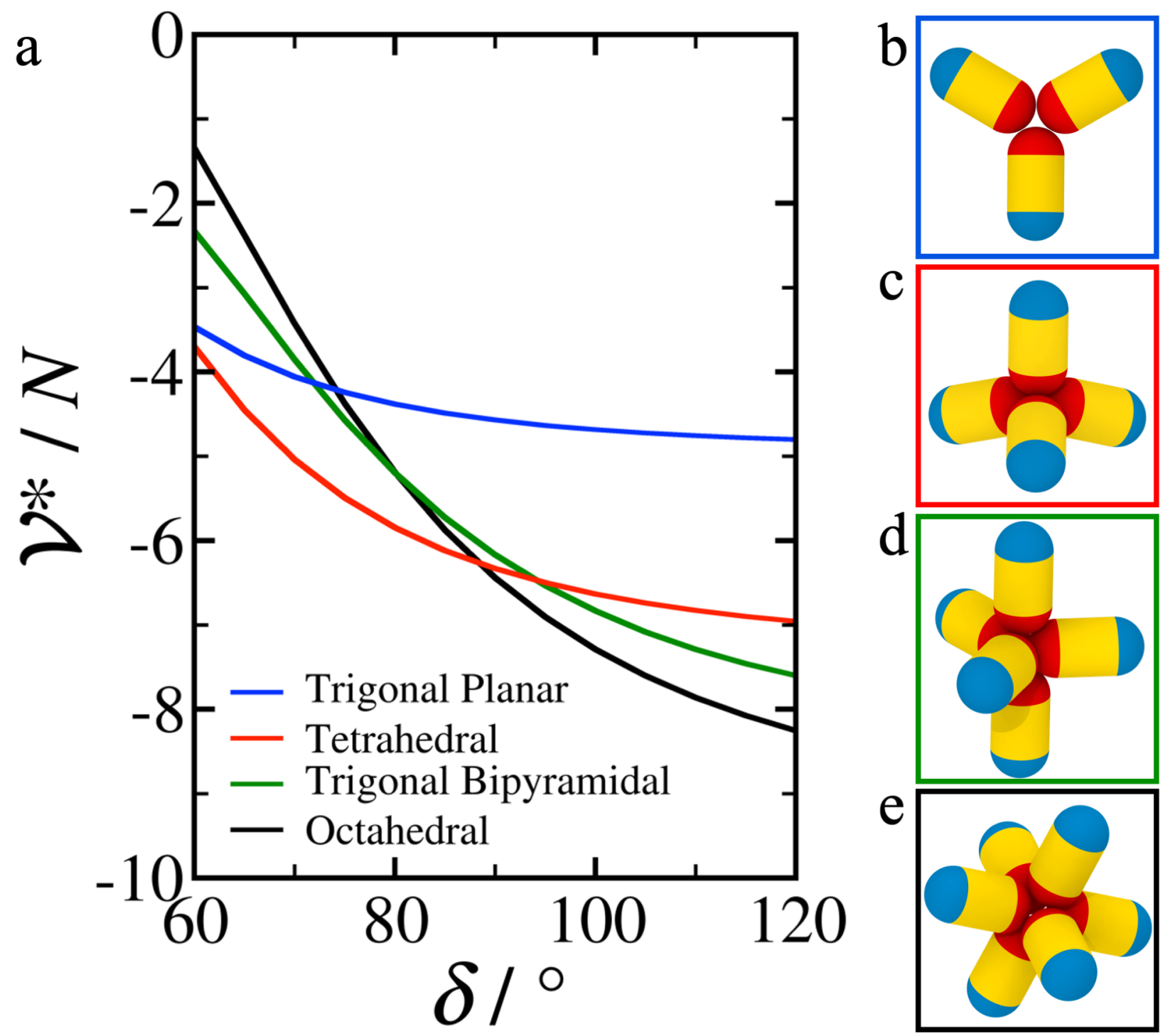

Supporting Figure 2: Energy per particle for different clusters of AB-triblock patchy rods as a function of the half-opening angle of the patches.

a) The potential energy per particle $\left(\mathcal{V}^{*} / N\right)$ is shown for trigonal planar, tetrahedral, trigonal bipyramidal and octahedral clusters as minima on the potential energy landscapes for ABtriblock patchy rods (TPRs) as a function of the half-opening angle $(\delta)$ of the patches. The potential energy is independent of the aspect ratio, as long as it is large enough to ensure that the particle can accommodate two non-overlapping patches. Snapshots of $\mathbf{b}$ ) trigonal planar, c) tetrahedral, d) trigonal bipyramidal and e) octahedral clusters of TPRs are also shown. 
a

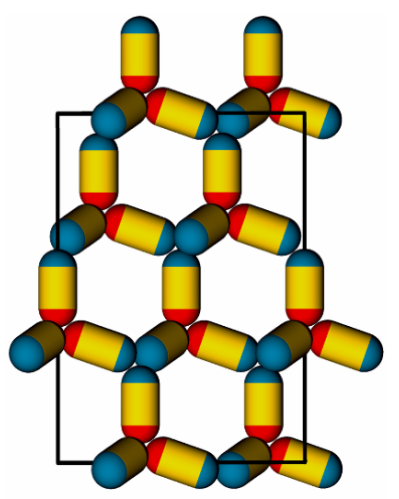

b
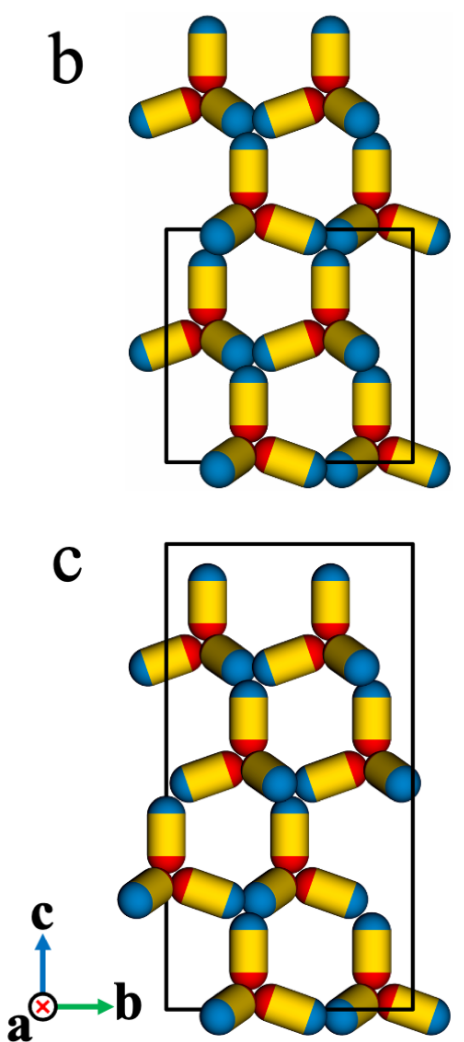
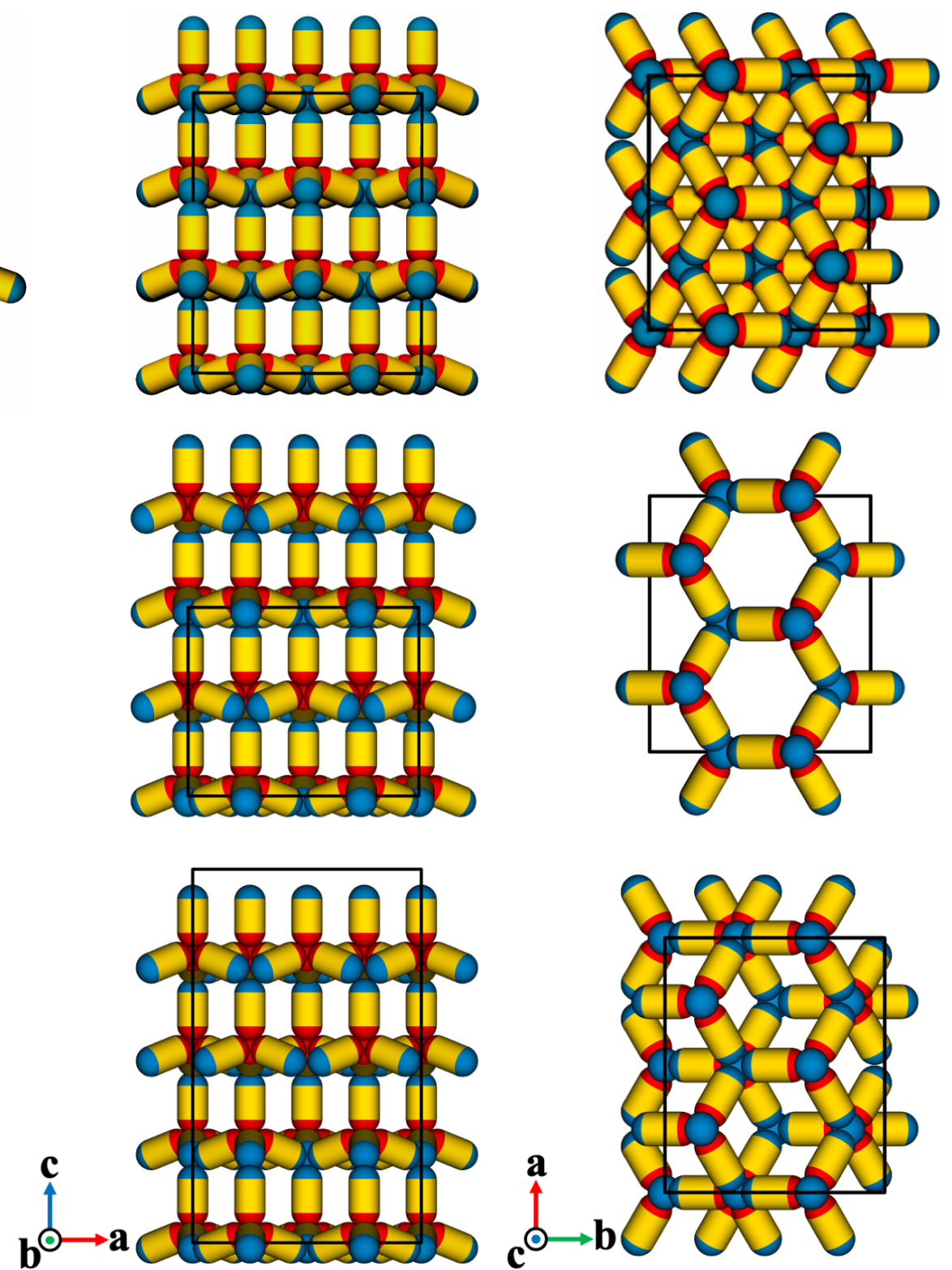
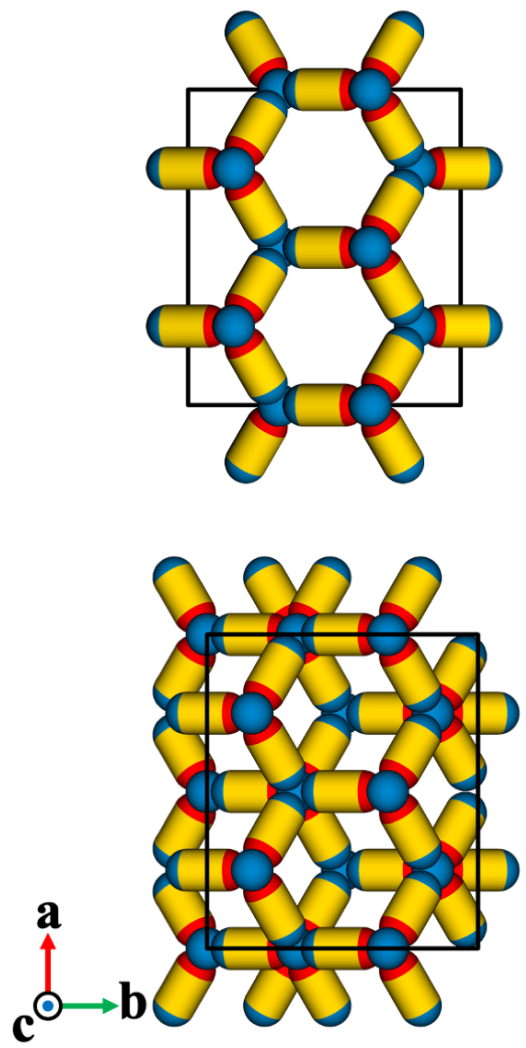

Supporting Figure 3: Views of different polymorphs of segmented rod-connected diamond (sRCD) crystal.

Orthorhombic unit cells of the (a) cubic, (b) hexagonal and (c) double-hexagonal sRCD structures are shown where the black lines outline the unit cell. The unit cell of the cubic polymorph is composed of three stacked layers of tetrahedra, where all adjacent layers are staggered relative to one another. The hexagonal sRCD structure has two layers of stacked tetrahedra, where all adjacent layers are eclipsed relative to one another. The double-hexagonal sRCD structure has four layers of stacked tetrahedra, where each layer has one adjacent staggered layer and one adjacent eclipsed layer. For example, the second layer in panel (c) is staggered relative to the 1st layer and eclipsed relative to the 3rd layer. 



Supporting Figure 4: Dependencies of the lattice energy and entropy of crystal structures of AB-triblock patchy rods (TPRs) on the patch half-opening angle and the aspect ratio.

a) Evolution of the lattice energy $\left(\mathcal{V}_{\text {Latt }}^{*}\right)$ per particle of the cubic and hexagonal segmented rod-connected diamond (sRCD) structures with the half-opening angle $(\delta=\alpha=\beta)$ of the attractive patches for AB-TPRs. Note that only one set of points are shown as $\mathcal{V}_{\text {Latt,c }}^{*}=$ $\mathcal{V}_{\text {Latt,h }}^{*}$. Additionally, the lattice energy does not change for different values of the aspect ratio $\left(L^{*}\right)$ of the TPRs. b) Dependence of the entropy $(S)$ for the c-sRCD and h-sRCD structures on the aspect ratio for three different half-opening angles of the attractive patches for AB-TPRs. The entropy was calculated in the $N V T$ ensemble using the Einstein crystal method for systems of $N=192 \mathrm{AB}-\mathrm{TPRs}$ at $T^{*}=0.1$ for $\delta=75^{\circ}, 80^{\circ}, 85^{\circ}$. 

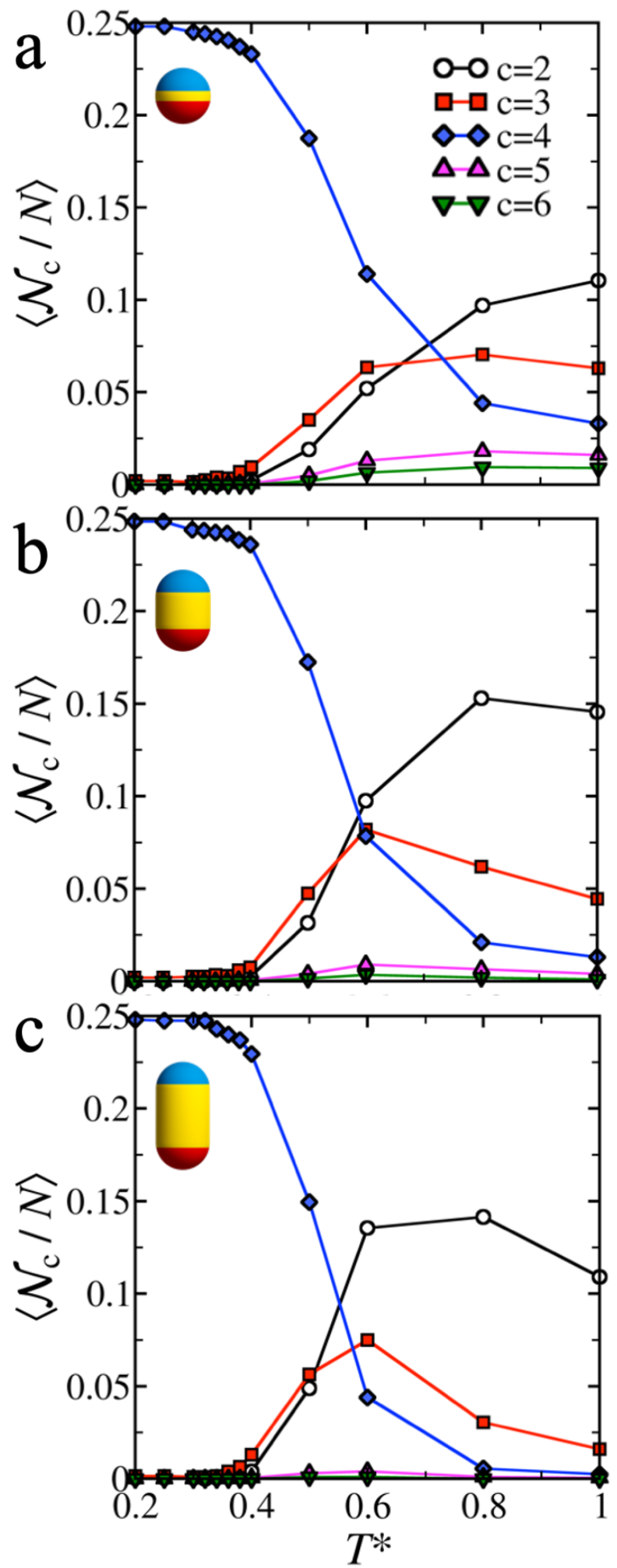

Supporting Figure 5: Evolution of cluster distributions with temperature in systems of AB-triblock patchy rods for different aspect ratios.

Evolution of the average number of clusters $\mathcal{N}_{\mathrm{c}}$ of size $c=2,3,4,5,6$ with temperature $T^{*}$ along a series of $N V T$ simulations for systems of $N=2000$ AB-TPRs with $\alpha=\beta=80^{\circ}$ for a) $\left.L^{*}=0, \rho^{*}=0.674 ; \mathbf{b}\right) L^{*}=0.5, \rho^{*}=0.241$; c) $L^{*}=1, \rho^{*}=0.11$. The insets show the corresponding TPRs. 

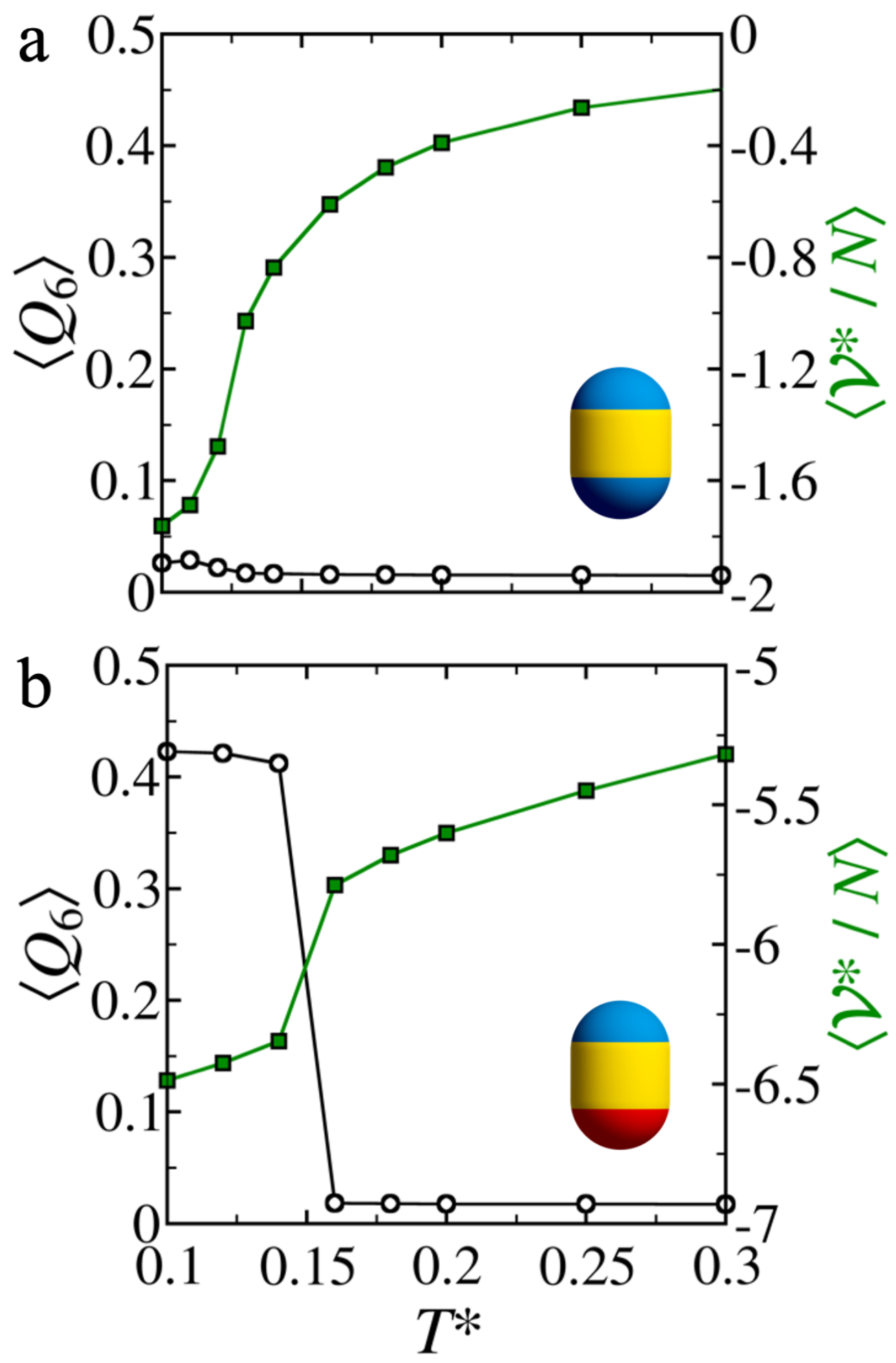

Supporting Figure 6: Promotion of crystallization by hierarchical self-assembly strategy. Evolution of the average energy per particle $\mathcal{V}^{*} / N$ and the global bondorientational order parameter $Q_{6}$ with temperature $T^{*}$ for the (a) AA-system and (b) ABsystem with $L^{\star}=0.5, N=2000$ and $\rho^{*}=0.241$. The insets show the corresponding designer triblock patchy rods. 

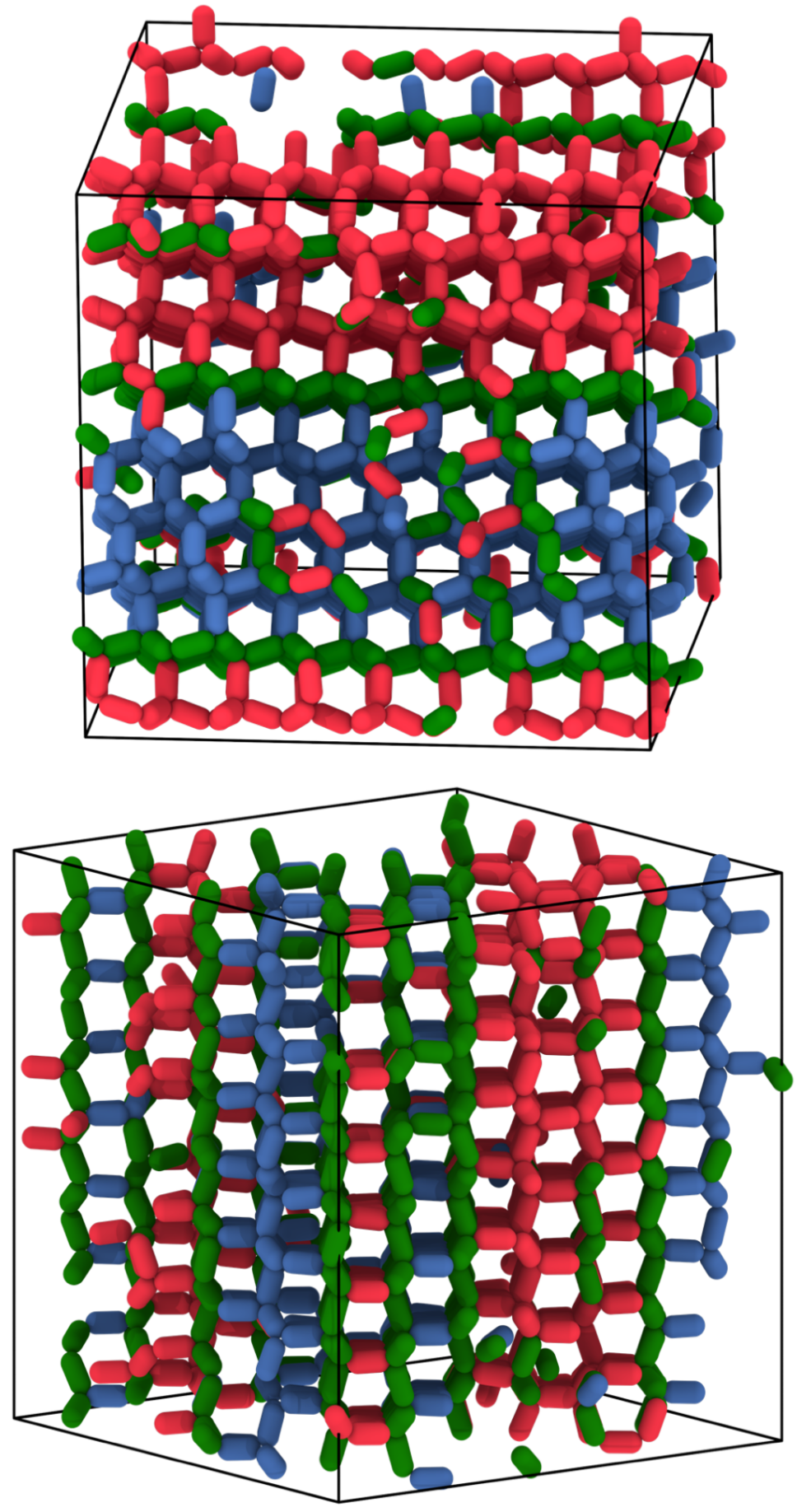

cubic Ohexagonal Ointerfacial

Supporting Figure 7: Local environments of particles in two representative crystalline configurations sampled in Monte Carlo simulations of the AB-triblock patchy rod (TPR) system.

The configurations were obtained for a system of $N=2000$ AB-TPRs with $L^{*}=0.5$ at $T^{*}=0.1$ and $\rho^{*}=0.241$. Here the particles are colored blue, red and green according to whether they are in locally cubic, hexagonal and interfacial environments, respectively. 

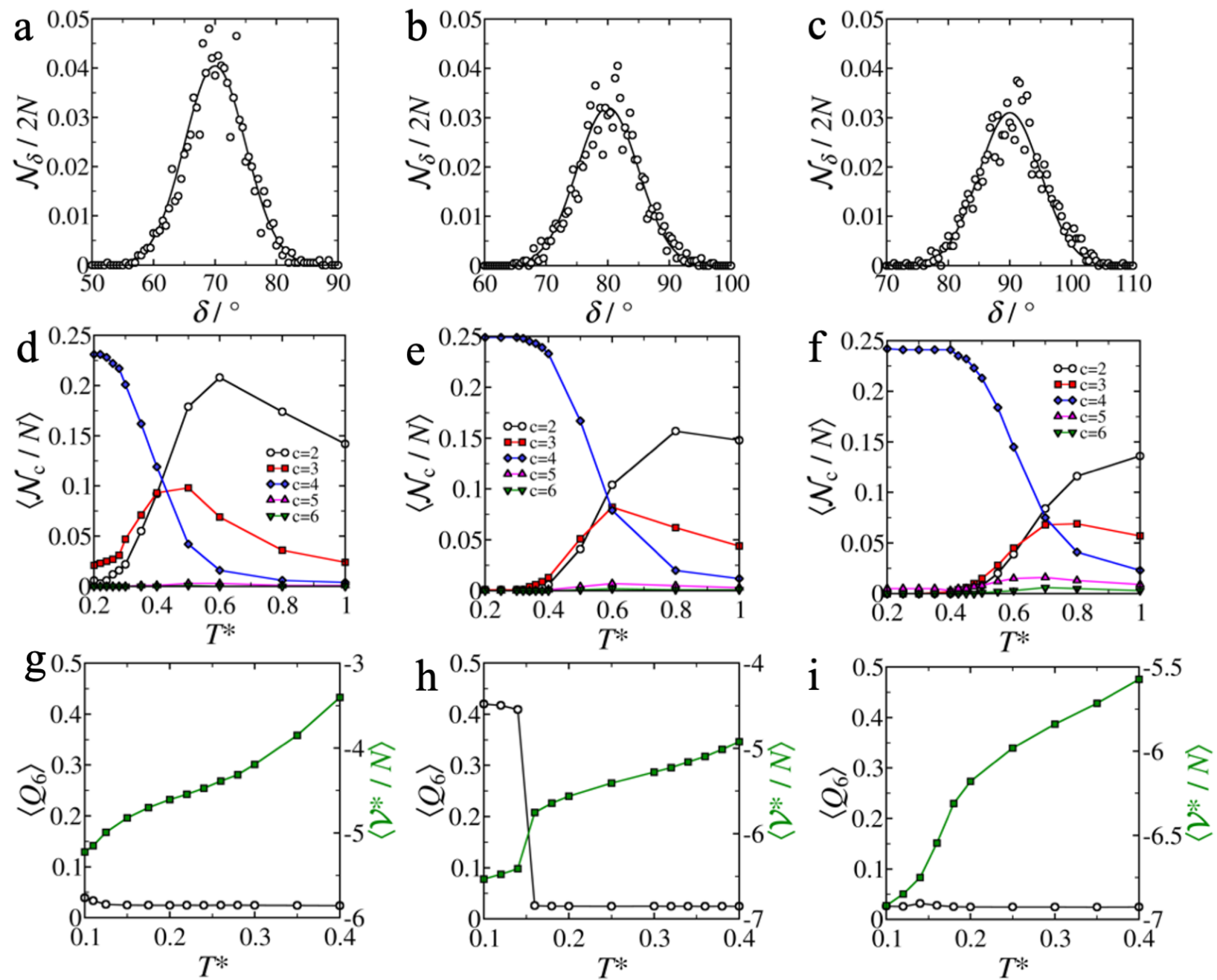

Supporting Figure 8: Self-assembly of AB-triblock patchy rods (TPRs) with polydisperse patch half-opening angles.

Data are presented for three different systems of $N=1000$ AB-TPRs with the aspect ratio $L^{*}=0.5$ and polydisperse patch half-angles $(\delta)$ having different values of the mean $\bar{\delta}$ but the same standard deviation $\sigma_{\delta}=5^{\circ}$ at $\rho^{*}=0.241$. a, $\mathbf{d}$ and $\mathbf{g}$ correspond to $\bar{\delta}=70^{\circ}$; $\bar{\delta}=80^{\circ}$ for $\mathbf{b}$, e and $\mathbf{h} ; \bar{\delta}=90^{\circ}$ for $\mathbf{c}, \mathbf{f}$ and i. (a-c) show the distributions of patchhalf-angles with a fit to a Gaussian distribution with mean $\bar{\delta}$ and standard deviation $\sigma_{\delta}$ for the three systems; (d-f) show the evolution of the average number of clusters $\left(\mathcal{N}_{\mathrm{c}}\right)$ of size $\mathrm{c}=2,3,4,5,6$ through the first stage of assembly in these systems; (g-i) show the evolution of the average energy $\left(\mathcal{V}^{*}\right)$ per particle and the global bond-orientational order parameter $\left(Q_{6}\right)$ at low temperatures for these systems. 



Supporting Figure 9: Snapshots of low-temperature configurations in systems of AB-triblock patchy rods (TPRs) with polydisperse patch half-opening angles. The configurations were obtained from systems of $N=1000$ AB-TPRs with $L^{*}=0.5$ at $T^{*}=0.1$ and $\rho^{*}=0.241$ with the patch half-angles $(\delta)$ having mean values: (a) $\bar{\delta}=70^{\circ},(\mathbf{b})$ $\bar{\delta}=80^{\circ}$, (c) $\bar{\delta}=90^{\circ}$, and the same standard deviation $\sigma_{\delta}=5^{\circ}$. The expanded view in panel (c) highlights the distorted trigonal bipyramidal motifs formed by the system of AB-TPRs with $\bar{\delta}=90^{\circ}$ following the first-stage of assembly. These distorted trigonal bipyramidal motifs can be considered as being formed via vertex-face bonds between the tetrahedral clusters that are facilitated by patch A-patch B interactions. Note that patch half-angles are not drawn to scale here. 



Supporting Figure 10: Scenarios with 5- and 6-member rings in AA-, AB- and AA'-triblock patchy rods systems.

(a) Representative scenarios with 5- and 6-member rings, which can form via identical patchpatch interactions in the AA-system. (b) Representative scenarios with 5- and 6-member rings formed in the AB-system that incorporates a hierarchy of patch-patch interactions. The formation of an AB-bond is essential for the formation of a 5-member ring as opposed to a 6-member ring; an A-B bond is possible to form during the second stage of assembly when A-patches are available to bond because of incomplete or incorrect first-stage of assembly. (c) Contrasting scenarios with 5- and 6-member rings in the $\mathrm{AA}^{\prime}$-system with specificity of patch-patch interactions such that there is no thermodynamic driving force for the formation of an $\mathrm{AA}^{\prime}$ bond. In this case, a 5-member ring is ruled out as opposed to a 6-member ring. 

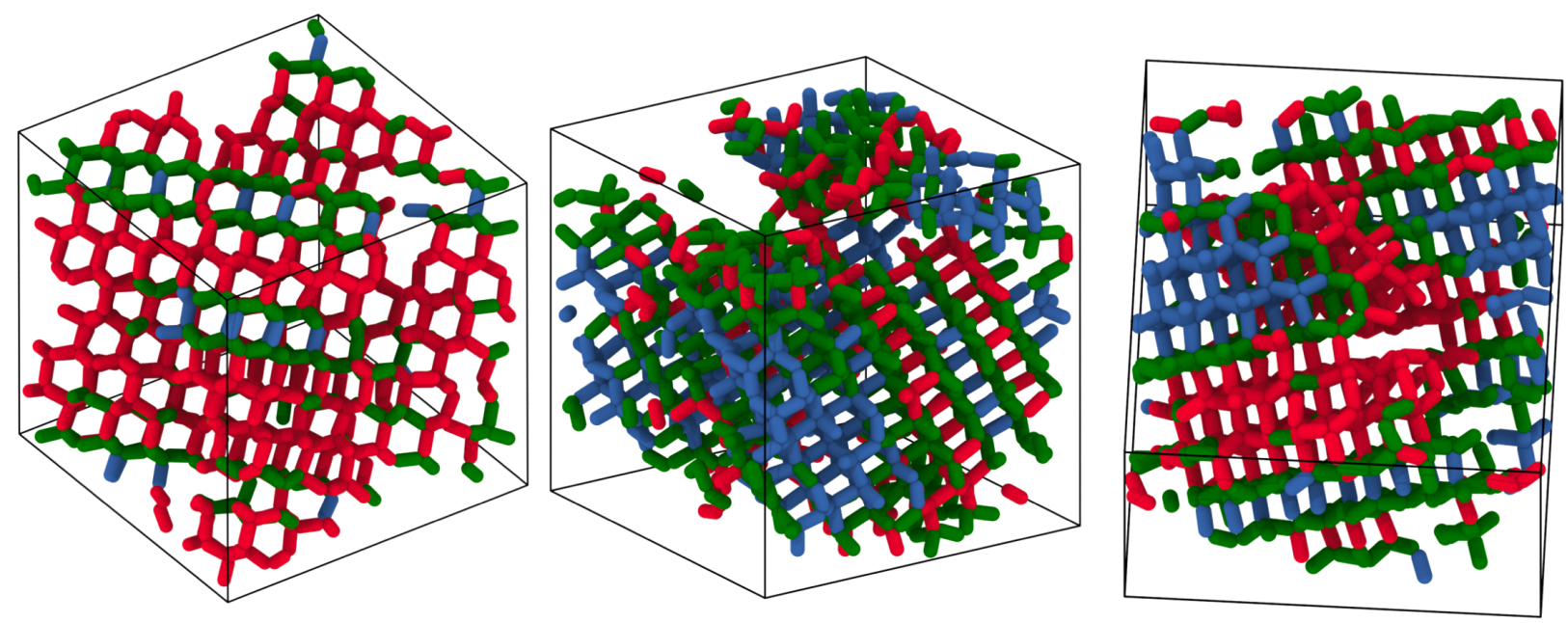

\section{Ocubic Ohexagonal Ointerfacial}

Supporting Figure 11: Local environments of particles in three representative crystalline configurations sampled in Monte Carlo simulations of the $\mathrm{AA}^{\prime}$ triblock patchy rod (TPR) system.

The configurations were obtained for a system of $N=2000 \mathrm{AA}^{\prime}$ TPRs with $L^{*}=0.5$ at $T^{*}=0.118$ and $\rho^{*}=0.241$. Here the particles are colored blue, red and green according to whether they are in locally cubic, hexagonal and interfacial environments, respectively. 

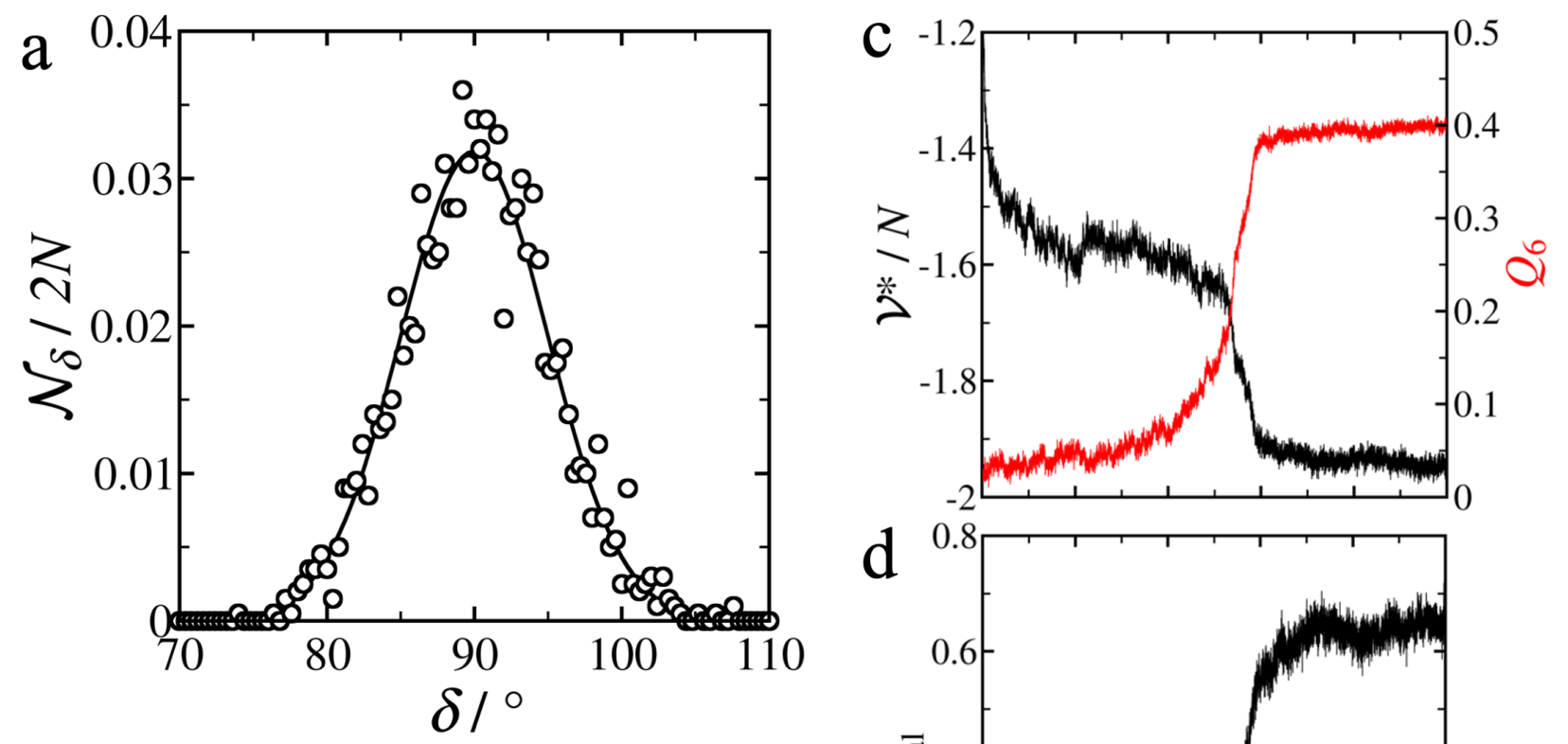

b
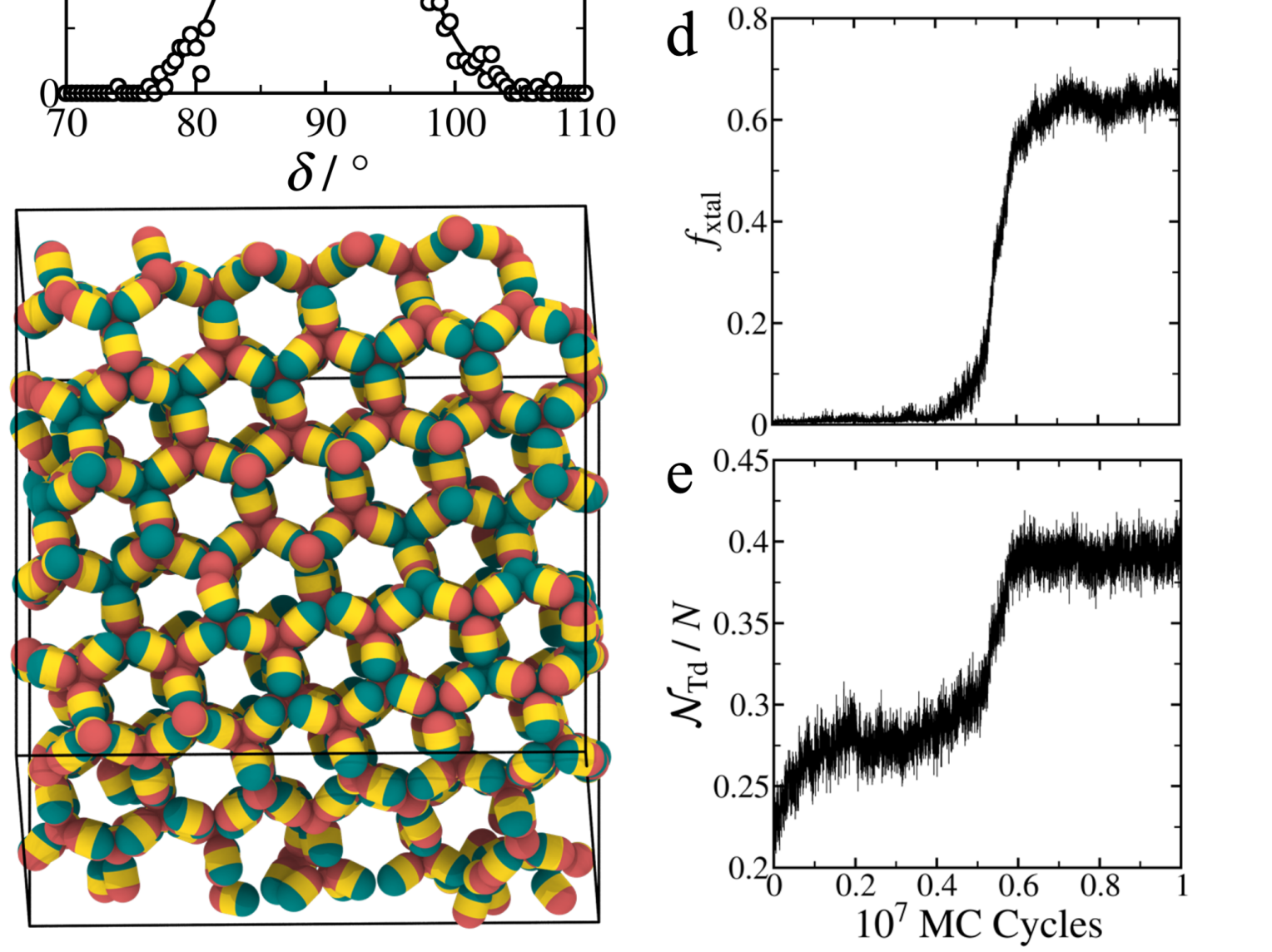

Supporting Figure 12: Nucleation of AA'-triblock patchy rods (TPRs) with polydisperse patch half-opening angles.

Crystallization is observed at the density $\rho^{*}=0.241$ and temperature $T^{*}=0.13$ in Monte Carlo simulations of a system of $N=1000 \mathrm{AA}^{\prime}$-TPRs with the aspect ratio $L^{*}=0.5$ and polydisperse patch half-opening angles with the patch half-angles $(\delta)$ having mean value of $\bar{\delta}=90^{\circ}$ and standard deviation $\sigma_{\delta}=5^{\circ}$. (a) Distribution of patch-half-angles with a fit to a Gaussian distribution with mean $\bar{\delta}$ and standard deviation $\sigma_{\delta}$; (b) representative snapshot of the system following crystallization. Here, patch half-angles are not drawn to scale. (c) evolution of the energy $\left(\mathcal{V}^{*}\right)$ per particle and the global bond-orientational order parameter $\left(Q_{6}\right) ;(\mathbf{d})$ the fraction of particles in the largest crystalline cluster $\left(f_{\mathrm{xtal}}\right)$; $(\mathbf{e})$ the total number of tetrahedra $\left(\mathcal{N}_{\mathrm{Td}}\right)$. 

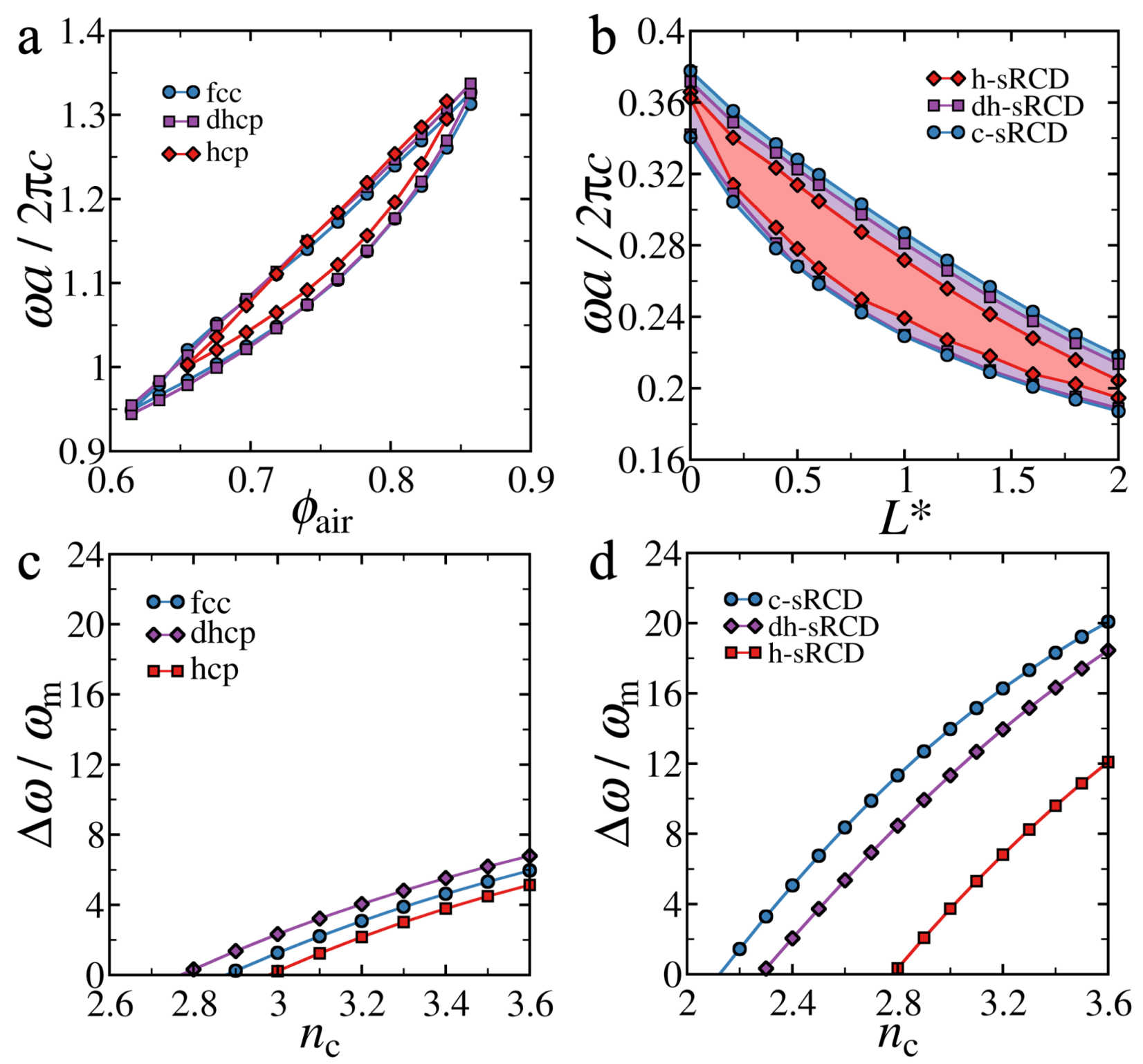

Supporting Figure 13: Comparison of photonic band gaps (PBGs) in inverse opal and segmented rod-connected diamond (sRCD) structures.

Photonic bandgap map with refractive index contrast of $n_{c}=3.6$, where the lower-frequency line corresponds to the dielectric band of the PBG and the higher-frequency line to the air band, for (a) inverse opal and (b) sRCD structures. The frequencies in the photonic bandgap maps for inverse opals are presented as a function of the volume fraction for the air spheres $\left(\phi_{\text {air }}\right)$, while for s-RCD structures they are presented as a function of the aspect ratio $L^{*}$. We take $a$ to be the edge length of the ideal primitive unit cell for the c-sRCD structure with $L^{*}=0(a \approx 2 \sigma)$ as determined by global optimization. Evolution of the relative gap width $\Delta \omega / \omega_{m}$, expressed as a percentage, with $n_{c}$ for the (c) inverse opal structures with $\phi_{\text {air }}=0.74$ and $(\mathbf{d})$ s-RCD structures with $L^{*}=0.5$ and density of $\rho^{*}=0.246$. We observe that, in both the inverse opal and sRCD structures, the cubic and hexagonal polymorphs possess PBGs with overlapping frequencies. We note that their respective stacking hybrids (dhcp and dh-sRCD) also possess a PBG in the same frequency region. 


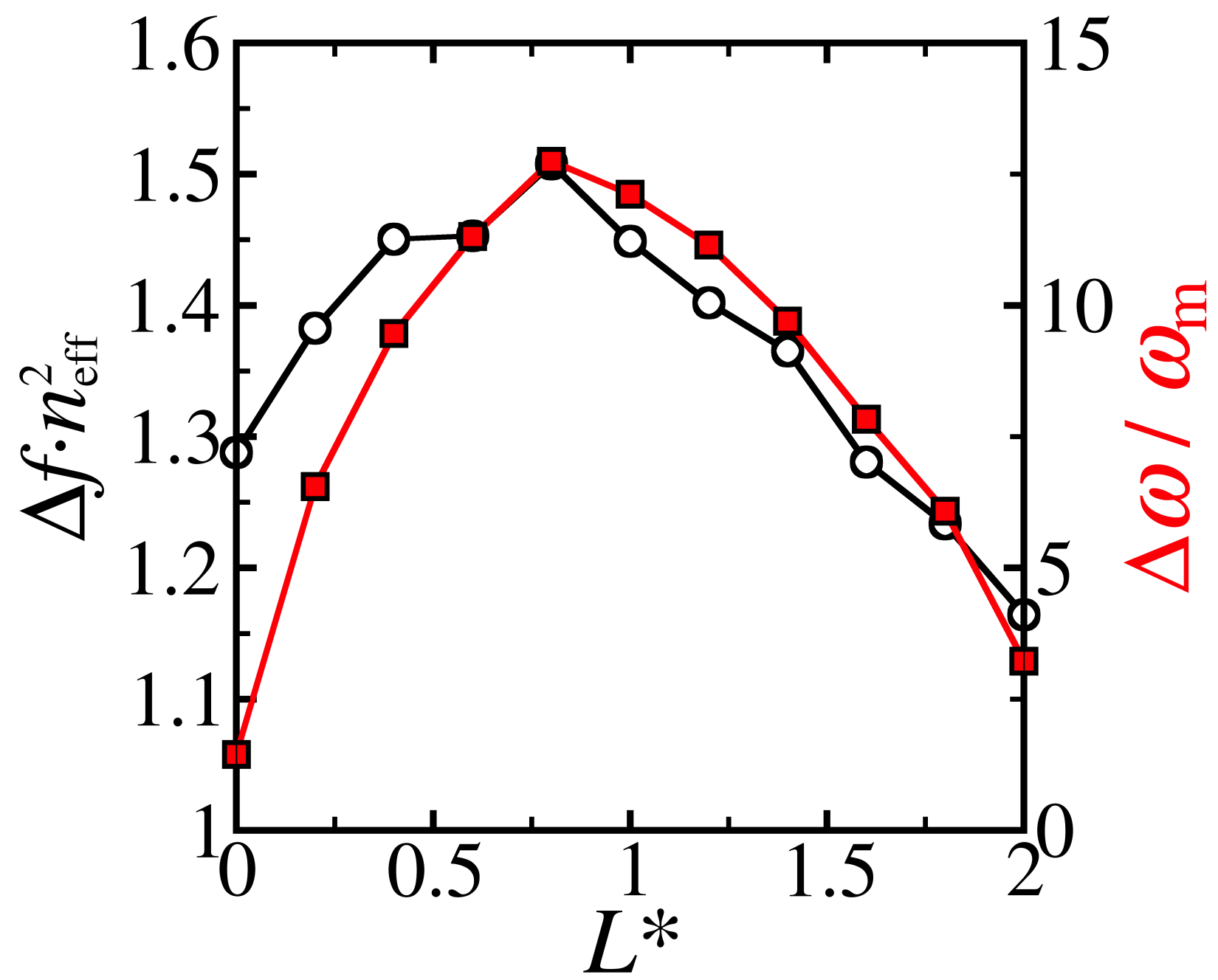

Supporting Figure 14: Aspect ratio dependence of the photonic band gap in the hexagonal segmented rod-connected diamond (h-sRCD) structure and its correspondence with a factor.

Evolution of the product $\Delta f \cdot n_{\text {eff }}^{2}$ with the aspect rato $L^{\star}$ for h-sRCD corresponding to the refractive index contrast $n_{c}=3.6$, where $\Delta f$ is the difference in concentration factor between the top of the dielectric band and bottom of the air band $\Delta f=f_{\text {diel }}-f_{\text {air }}$ and $n_{\text {eff }}$ is the mean refractive-index $n_{\text {eff }}$ of the crystal. The peak in $\Delta f \cdot n_{\text {eff }}^{2}$ coincides with the peak in the relative gap width of the photonic band gap $(\mathrm{PBG})$, expressed as the percentage band gap to mid gap ratio $\Delta \omega / \omega_{m}$. 

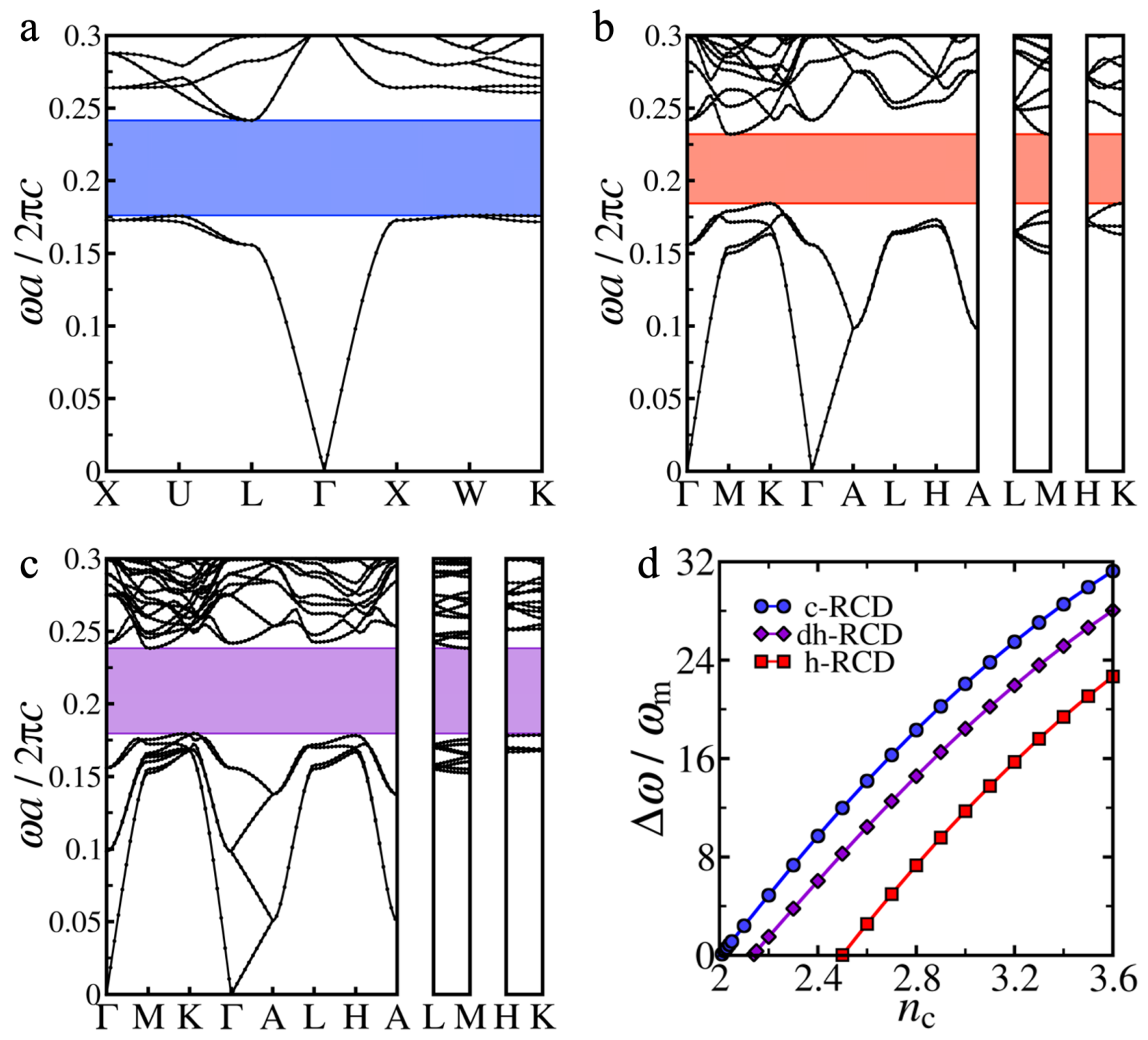

Supporting Figure 15: Photonic band structures for rod-connected diamond crystals.

Photonic band structures for the (a) cubic, (b) double-hexagonal and (c) hexagonal rodconnected diamond structures for a refractive-index contrast of $n_{c}=\sqrt{13}$ with $L^{*}=1.2$ and an effective particle diameter of $1.2 \sigma$. The relative gap width of the complete PBG for the c-RCD structure converges on the previously quoted value of $31 \%$ at $n_{c}=\sqrt{13}$. $^{9110}$ Moreover, the dh-RCD structure assumes the "near-champion" status as it supports a relative gap width of $28 \%$ at $n_{c}=\sqrt{13}$, the same as the single-network gyroid structure. $\frac{10}{10}$ This is surprising, as only crystal structures with cubic symmetry have hitherto been sought after for their well-documented ability to support such a wide complete PBG. ${ }^{11}$ (d) Evolution of the relative gap width $\Delta \omega / \omega_{m}$, expressed as a percentage, with the refractive index contrast $n_{c}$ for the cubic, double-hexagonal and hexagonal rod-connected diamond structures. 


\section{Supporting Movies}

Supporting Movie 1: Growth of the largest crystalline cluster in the AA'-triblock patchy rod system

Visualization of the growth of the largest crystalline cluster in a system of $N=2000 \mathrm{AA}^{\prime}$ triblock patchy rods (TPRs) at $\rho^{*}=0.241$ and $T^{*}=0.118$ upon cooling from $T^{*}=1$. The

formation and dissolution of a crystalline cluster continues until a critical nucleus is formed, beyond which a rapid growth of the crystal takes place. The emergence of local tetrahedral ordering in the largest crystalline cluster in its early stage is apparent. The growth of the nucleus is marked by the emergence of the rings.

\section{Supporting Movie 2: Evolution of tetrahedral clusters in the AA'-triblock patchy rod system \\ Visualization of the tetrahedral clusters formed in a system of $N=2000 \mathrm{AA}^{\prime}$-triblock patchy rods (TPRs) at $\rho^{*}=0.241$ and $T^{*}=0.118$ upon cooling from $T^{*}=1$. A reduced represen- tation is followed here with the tetrahedral clusters shown as faceted tetrahedra, where each vertex of a tetrahedron corresponds to the center of a patch on a triblock patchy rod particle. The tetrahedra are colored red if they are formed by A-patches and teal if they are formed by B-patches. The movie shows that the system evolves through a fluid phase of tetrahedral clusters before crystalline order emerges marked by the formation of the rings. Note that frequencies at which the frames are shown in Supporting Movies 1 and 2 are different.}




\section{References}

1. Haxton, T. K.; Whitelam, S. Do Hierarchical Structures Assemble Best via Hierarchical Pathways? Soft Matter 2013, 9, 6851-6861.

2. Morphew, D.; Shaw, J.; Avins, C.; Chakrabarti, D. Programming Hierarchical SelfAssembly of Patchy Particles into Colloidal Crystals via Colloidal Molecules. ACS Nano 2018, 12, 2355-2364.

3. Rao, A. B.; Shaw, J.; Neophytou, A.; Morphew, D.; Sciortino, F.; Johnston, R. L.; Chakrabarti, D. Leveraging Hierarchical Self-Assembly Pathways for Realizing Colloidal Photonic Crystals. ACS Nano 2020, 14, 5348-5349.

4. Chen, Q.; Whitmer, J. K.; Jiang, S.; Bae, S. C.; Luijten, E.; Granick, S. Supracolloidal Reaction Kinetics of Janus Spheres. Science 2011, 331, 199-202.

5. Chaudhary, K.; Chen, Q.; Juárez, J. J.; Granick, S.; Lewis, J. A. Janus Colloidal Matchsticks. J. Am. Chem. Soc. 2012, 134, 12901-12903.

6. Oh, J. S.; Lee, S.; Glotzer, S. C.; Yi, G.-R.; Pine, D. J. Colloidal Fibers and Rings by Cooperative Assembly. Nat. Commun. 2019, 10, 3936.

7. Kern, N.; Frenkel, D. Fluid-Fluid Coexistence in Colloidal Systems with Short-Ranged Strongly Directional Attraction. J. Chem. Phys. 2003, 118, 9882-9889.

8. Chen, Q.; Bae, S. C.; Granick, S. Staged Self-Assembly of Colloidal Metastructures. J. Am. Chem. Soc. 2012, 134, 11080-11083.

9. Ho, K. M.; Chan, C. T.; Soukoulis, C. M. Existence of a Photonic Gap in Periodic Dielectric Structures. Phys. Rev. Lett. 1990, 65, 3152-3155.

10. Sellers, S. R.; Man, W.; Sahba, S.; Florescu, M. Local Self-Uniformity in Photonic Networks. Nat. Commun. 2017, 8, 14439. 
11. Men, H.; Lee, K. Y. K.; Freund, R. M.; Peraire, J.; Johnson, S. G. Robust Topology Optimization of Three-Dimensional Photonic-Crystal Band-Gap Structures. Opt. Express 2014, 22, 22632-22648. 